\title{
D-metric Spaces and Composition Operators Between Hyperbolic Weighted Family of Function Spaces
}

\author{
A. KAMAL $^{1}$ AND T.I.YASSEN ${ }^{2}$ \\ ${ }^{1}$ Port Said University, Faculty of Science, \\ Department of Mathematics and Computer Science, \\ Port Said, Egypt. \\ 2 The Higher Engineering Institute in Al-Minya (EST-Minya) \\ Minya, Egypt. \\ alaa_mohamed1@yahoo.com, taha_hmour@yahoo.com
}

\begin{abstract}
The aim of this paper is to introduce new hyperbolic classes of functions, which will be called $\mathcal{B}_{\alpha, \log }^{*}$ and $F_{\log }^{*}(p, q, s)$ classes. Furthermore, we introduce $D$-metrics space in the hyperbolic type classes $\mathcal{B}_{\alpha, \log }^{*}$ and $F_{\log }^{*}(p, q, s)$. These classes are shown to be complete metric spaces with respect to the corresponding metrics. Moreover, necessary and sufficient conditions are given for the composition operator $C_{\phi}$ to be bounded and compact from $\mathcal{B}_{\alpha, \log }^{*}$ to $F_{\log }^{*}(p, q, s)$ spaces.

\section{RESUMEN}

El objetivo de este artículo es introducir nuevas clases hiperbólicas de funciones, que serán llamadas clases $\mathcal{B}_{\alpha, \log }^{*}$ y $F_{\log }^{*}(p, q, s)$. A continuación, introducimos $D$-espacios métricos en las clases de tipo hiperbólicas $\mathcal{B}_{\alpha, \log }^{*}$ y $F_{\log }^{*}(p, q, s)$. Mostramos que estas clases son espacios métricos completos con respecto a las métricas correspondientes. Más aún, damos condiciones necesarias y suficientes para que el operador composición $C_{\phi}$ sea acotado y compacto desde el espacio $\mathcal{B}_{\alpha, \log }^{*}$ a $F_{\log }^{*}(p, q, s)$.
\end{abstract}

Keywords and Phrases: D-metric spaces, Logarithmic hyperbolic classes, Composition operators.

2020 AMS Mathematics Subject Classification: 47B38, 46E15. 


\section{Introduction}

Let $\phi$ be an analytic self-map of the open unit disk $\mathbb{D}=\{z \in \mathbb{C}:|z|<1\}$ in the complex plane $\mathbb{C}$ and let $\partial \mathbb{D}$ be its boundary. Let $H(\mathbb{D})$ denote the space of all analytic functions in $\mathbb{D}$ and let $B(\mathbb{D})$ be the subset of $H(\mathbb{D})$ consisting of those $f \in H(\mathbb{D})$ for which $|f(z)|<1$ for all $z \in \mathbb{D}$.

Let the Green's function of $\mathbb{D}$ be defined as $g(z, a)=\log \frac{1}{\left|\varphi_{a}(z)\right|}$, where $\varphi_{a}(z)=\frac{a-z}{1-\bar{a} z}$ is the Möbius transformation related to the point $a \in \mathbb{D}$.

A linear composition operator $C_{\phi}$ is defined by $C_{\phi}(f)=(f \circ \phi)$ for $f$ in the set $H(\mathbb{D})$ of analyticfunctions on $\mathbb{D}$ (see [9]). A function $f \in B(\mathbb{D})$ belongs to $\alpha$-Bloch space $\mathcal{B}_{\alpha}, 0<\alpha<\infty$, if

$$
\|f\|_{\mathcal{B}_{\alpha}}=\sup _{z \in \mathbb{D}}(1-|z|)^{\alpha}\left|f^{\prime}(z)\right|<\infty
$$

The little $\alpha$-Bloch space $\mathcal{B}_{\alpha, 0}$ consisting of all $f \in \mathcal{B}_{\alpha}$ so that

$$
\lim _{|z| \rightarrow 1^{-}}\left(1-|z|^{2}\right)\left|f^{\prime}(z)\right|=0
$$

Definition 1. [15] For an analytic function $f$ on $\mathbb{D}$ and $0<\alpha<\infty$, if

$$
\|f\|_{\mathcal{B}_{\log }^{\alpha}}=\sup _{z \in \mathbb{D}}\left(1-|z|^{2}\right)^{\alpha}\left|f^{\prime}(z)\right|\left(\log \frac{2}{1-|z|^{2}}\right)<\infty
$$

then, $f$ belongs to the weighted $\alpha$-Bloch spaces $\mathcal{B}_{\log }^{\alpha}$.

If $\alpha=1$, the weighted Bloch space $\mathcal{B}_{\log }$ is the set for all analytic functions $f$ in $\mathbb{D}$ for which $\|f\|_{\mathcal{B}_{\log }}<\infty$.

The expression $\|f\|_{\mathcal{B}_{\log }}$ defines a seminorm while the norm is defined by

$$
\|f\|_{\mathcal{B}_{\log }}=|f(0)|+\|f\|_{\mathcal{B}_{\log }} .
$$

Definition 2. 14 For $0<p, s<\infty,-2<q<\infty$ and $q+s>-1$, a function $f \in H(\mathbb{D})$ is in $F(p, q, s)$, if

$$
\sup _{a \in \mathbb{D}} \int_{\mathbb{D}}\left|f^{\prime}(z)\right|^{p}\left(1-|z|^{2}\right)^{q} g^{s}(z, a) d A(z)<\infty .
$$

Moreover, if

$$
\lim _{|a| \rightarrow 1^{-}} \int_{\mathbb{D}}\left|f^{\prime}(z)\right|^{p}\left(1-|z|^{2}\right)^{q} g^{s}(z, a) d A(z)=0,
$$

then $f \in F_{0}(p, q, s)$.

El-Sayed and Bakhit [5] gave the following definition: 
Definition 3. For $0<p, s<\infty,-2<q<\infty$ and $q+s>-1$, a function $f \in H(\mathbb{D})$ is said to belong to $F_{\log }(p, q, s)$, if

$$
\sup _{I \subset \partial \mathbb{D}} \frac{\left(\log \frac{2}{|I|}\right)^{p}}{|I|^{s}} \int_{S(I)}\left|f^{\prime}(z)\right|^{p}\left(1-|z|^{2}\right)^{q}\left(\log \frac{1}{|z|}\right)^{s} d A(z)<\infty .
$$

Where $|I|$ denotes the arc length of $I \subset \partial \mathbb{D}$ and $S(I)$ is the Carleson box defined by (see [8, [6])

$$
S(I)=\left\{z \in \mathbb{D}: 1-|I|<|z|<1, \frac{z}{|z|} \in|I|\right\} .
$$

The interest in the $F_{\log }(p, q, s)$-spaces rises from the fact that they cover some well known function spaces. It is immediate that $F_{\log }(2,0,1)=B M O A_{\log }$ and $F_{\log }(2,0, p)=Q_{\log }^{p}$, where $0<p<\infty$.

\section{Preliminaries}

Definition 4. [11] The hyperbolic Bloch space $\mathcal{B}_{\alpha}^{*}$ is defined as

$$
\mathcal{B}_{\alpha}^{*}=\left\{f: f \in B(\mathbb{D}) \text { and } \sup _{z \in \mathbb{D}}\left(1-|z|^{2}\right)^{\alpha} f^{*}(z)<\infty\right\} .
$$

Denoting $f^{*}(z)=\frac{\left|f^{\prime}(z)\right|}{1-|f(z)|^{2}}$, the hyperbolic derivative of $f \in B(\mathbb{D}) .[7]$

The little hyperbolic Bloch space $\mathcal{B}_{\alpha, 0}^{*}$ is a subspace of $\mathcal{B}_{\alpha}^{*}$ consisting of all $f \in \mathcal{B}_{\alpha}^{*}$ so that

$$
\lim _{|z| \rightarrow 1^{-}}\left(1-|z|^{2}\right)^{\alpha} f^{*}(z)=0 .
$$

The space $\mathcal{B}_{\alpha}^{*}$ is Banach space with the norm defined as

$$
\|f\|_{\mathcal{B}_{\alpha}^{*}}=|f(0)|+\sup _{z \in \mathbb{D}}(1-|z|)^{\alpha}\left|f^{*}(z)\right| .
$$

Definition 5. For $0<p, s<\infty,-2<q<\infty, \alpha=\frac{q+2}{p}$ and $q+s>-1$, a function $f \in H(\mathbb{D})$ is said to belong to $F^{*}(p, q, s)$, if

$$
\sup _{a \in \mathbb{D}} \int_{\mathbb{D}}\left(f^{*}(z)\right)^{p}\left(1-|z|^{2}\right)^{\alpha p-2} g^{s}(z, a) d A(z)<\infty .
$$

Definition 6. For $f \in B(\mathbb{D})$ and $0<\alpha<\infty$, if

$$
\|f\|_{\mathcal{B}_{\alpha, \log }^{*}}=\sup _{z \in \mathbb{D}}\left(1-|z|^{2}\right)^{\alpha}\left(f^{*}(z)\right)\left(\log \frac{2}{1-|z|^{2}}\right)<\infty,
$$

then $f$ belongs to the $\mathcal{B}_{\alpha, \log }^{*}$. 
We must consider the following lemmas in our study:

Lemma 2.1. [12] Let $0<r \leq t \leq 1$, then

$$
\log \frac{1}{t} \leq \frac{1}{r}\left(1-t^{2}\right)
$$

Lemma 2.2. [12] Let $0 \leq k_{1}<\infty, 0 \leq k_{2}<\infty$, and $k_{1}-k_{2}>-1$, then

$$
C\left(k_{1}, k_{2}\right)=\int_{\mathbb{D}}\left(\log \frac{1}{|z|}\right)^{k_{1}}\left(1-|z|^{2}\right)^{-k_{2}} d A(z)<\infty .
$$

To study composition operators on $\mathcal{B}_{\alpha, \log }^{*}$ and $F_{\log }^{*}(p, q, s)$ spaces, we need to prove the following result:

Theorem 1. If $0<p<\infty, 1<s<\infty$ and $\alpha=\frac{q+2}{p}$ with $q+s>-1$. Then the following are equivalent:

(A) $f \in \mathcal{B}_{\alpha, \log }^{*}$

(B) $f \in F_{\log }^{*}(p, q, s)$.

(C) $\sup _{a \in \mathbb{D}}\left(\log \frac{2}{1-|a|^{2}}\right)^{p} \int_{\mathbb{D}}\left(f^{*}(z)\right)^{p}\left(1-|z|^{2}\right)^{\alpha p-2}\left(1-|\varphi(z)|^{2}\right)^{s} d A(z)<\infty$,

(D) $\sup _{a \in \mathbb{D}}\left(\log \frac{2}{1-|a|^{2}}\right)^{p} \int_{\mathbb{D}}\left(f^{*}(z)\right)^{p}\left(1-\left.|z|^{2}\right|^{\alpha p-2} g^{s}(z, a) d A(z)<\infty\right.$.

Proof. Let $0<p<\infty,-2<q<\infty, 1<s<\infty$ and $0<r<1$. By subharmonicity we have for an analytic function $g \in \mathbb{D}$ that

$$
|g(0)|^{p} \leq \frac{1}{\pi r^{2}} \int_{\mathbb{D}(0, r)}|g(w)|^{p} d A(w)
$$

For $a \in \mathbb{D}$, the substitution $z=\varphi_{a}(z)$ results in Jacobian change in measure given by

$$
d A(w)=\left|\varphi_{a}^{\prime}(z)\right|^{2} d A(z)
$$

For a Lebesgue integrable or a non-negative Lebesgue measurable function $f$ on $\mathbb{D}$, we thus have the following change of variable formula:

$$
\int_{\mathbb{D}(0, r)} f\left(\varphi_{a}(w)\right) d A(w)=\int_{\mathbb{D}(a, r)} f(z)\left|\varphi_{a}^{\prime}(z)\right|^{2} d A(z) .
$$

Let $g=\frac{f^{\prime} \circ \varphi_{a}}{1-\left|f \circ \varphi_{a}\right|^{2}}$ then we have

$$
\begin{aligned}
\left(\frac{\left|f^{\prime}(a)\right|}{1-|f(a)|^{2}}\right)^{p}=\left(f^{*}(a)\right)^{p} & \leq \frac{1}{\pi r^{2}} \int_{\mathbb{D}(0, r)}\left(\frac{\left|f^{\prime}\left(\varphi_{a}(w)\right)\right|}{1-\left|f\left(\varphi_{a}(w)\right)\right|^{2}}\right)^{p} d A(w) \\
& =\frac{1}{\pi r^{2}} \int_{\mathbb{D}(a, r)}\left(f^{*}(z)\right)^{p}\left|\varphi_{a}^{\prime}(z)\right|^{2} d A(z)
\end{aligned}
$$


Since

$$
\left|\varphi_{a}^{\prime}(z)\right|=\frac{1-\left|\varphi_{a}(z)\right|^{2}}{1-|z|^{2}}
$$

and

$$
\frac{1-\left|\varphi_{a}(z)\right|^{2}}{1-|z|^{2}} \leq \frac{4}{1-|a|^{2}} \quad a, z \in \mathbb{D}
$$

So we obtain that

$$
\left(f^{*}(a)\right)^{p} \leq \frac{16}{\pi r^{2}\left(1-|a|^{2}\right)^{2}} \int_{\mathbb{D}(a, r)}\left(f^{*}(z)\right)^{p} d A(z) .
$$

Again $f \in \mathcal{B}_{\alpha, \log }^{*}$, and $\left(1-|z|^{2}\right)^{2} \approx\left(1-|a|^{2}\right)^{2} \approx \mathbb{D}(a, r)$, for $z \in \mathbb{D}(a, r)$. Thus, we have

$$
\begin{aligned}
\left(\log \frac{2}{1-|a|^{2}}\right)^{p}\left(f^{*}(a)\right)^{p}\left(1-|a|^{2}\right)^{\alpha p} \\
\quad \leq \frac{16}{\pi r^{2}\left(1-|a|^{2}\right)^{2-\alpha p}} \times\left(\log \frac{2}{1-|a|^{2}}\right)^{p} \int_{\mathbb{D}(a, r)}\left(f^{*}(z)\right)^{p} d A(z) \\
\quad \leq \frac{16}{\pi r^{2}} \times\left(\log \frac{2}{1-|a|^{2}}\right)^{p} \int_{\mathbb{D}(a, r)}\left(f^{*}(z)\right)^{p}\left(1-|z|^{2}\right)^{\alpha p-2} d A(z) \\
\leq \frac{16}{\pi r^{2}} \times\left(\log \frac{2}{1-|a|^{2}}\right)^{p} \int_{\mathbb{D}(a, r)}\left(f^{*}(z)\right)^{p}\left(1-|z|^{2}\right)^{\alpha p-2} \times\left(\frac{1-\left|\varphi_{a}(z)\right|^{2}}{1-\left|\varphi_{a}(z)\right|^{2}}\right)^{s} d A(z) \\
\quad \leq \frac{16}{\pi r^{2}\left(1-r^{2}\right)^{s}} \times\left(\log \frac{2}{1-|a|^{2}}\right)^{p} \int_{\mathbb{D}(a, r)}\left(f^{*}(z)\right)^{p}\left(1-|z|^{2}\right)^{\alpha p-2}\left(1-\left|\varphi_{a}(z)\right|^{2}\right)^{s} d A(z) \\
\quad \leq M(r) \times\left(\log \frac{2}{1-|a|^{2}}\right)^{p} \int_{\mathbb{D}(a, r)}\left(f^{*}(z)\right)^{p}\left(1-|z|^{2}\right)^{\alpha p-2}\left(1-\left|\varphi_{a}^{\prime}(z)\right|^{2}\right)^{s} d A(z) .
\end{aligned}
$$

Where $M(r)$ is a constant depending on $r$. Thus, the quantity (A) is less than or equal to constant times the quantity $(\mathrm{C})$.

From the fact

$$
\left(1-\left|\varphi_{a}(z)\right|^{2}\right) \leq 2 \log \frac{1}{\left|\varphi_{a}(z)\right|}=2 g(z, a) \quad \text { for } a, z \in \mathbb{D},
$$

we have

$$
\begin{aligned}
& \left(\log \frac{2}{1-|a|^{2}}\right)^{p} \int_{\mathbb{D}(a, r)}\left(f^{*}(z)\right)^{p}\left(1-|z|^{2}\right)^{\alpha p-2}\left(1-\left|\varphi_{a}(z)\right|^{2}\right)^{s} d A(z) \\
\leq & \left(\log \frac{2}{1-|a|^{2}}\right)^{p} \int_{\mathbb{D}(a, r)}\left(f^{*}(z)\right)^{p}\left(1-|z|^{2}\right)^{\alpha p-2} g^{s}(z, a) d A(z) .
\end{aligned}
$$

Hence, the quantity (C) is less than or equal to a constant times (D). By taking $\alpha=\frac{q+2}{p}$, it follows $f \in F_{\log }^{*}(p, q, s)$. Thus, the quantity (C) is less than or equal to a constant times the quantity (B). 
Finally, from the following inequality, let $z=\varphi_{a}(w)$ then $w=\varphi_{a}(z)$. Hence,

$$
\begin{aligned}
& \left(\log \frac{2}{1-|a|^{2}}\right)^{p} \int_{\mathbb{D}}\left(f^{*}\left(\varphi_{a}(w)\right)\right)^{p}\left(1-\left|\varphi_{a}(w)\right|^{2}\right)^{\alpha p-2}\left(\log \frac{1}{|w|}\right)^{s}\left|\varphi_{a}^{\prime}(w)\right|^{2} d A(w) \\
= & \left(\log \frac{2}{1-|a|^{2}}\right)^{p} \int_{\mathbb{D}}\left(f^{*}\left(\varphi_{a}(w)\right)\right)^{p}\left(1-\left|\varphi_{a}(w)\right|^{2}\right)^{\alpha p}\left(\log \frac{1}{|w|}\right)^{s} \frac{\left|\varphi_{a}^{\prime}(w)\right|^{2}}{\left(1-\left|\varphi_{a}(w)\right|^{2}\right)^{2}} d A(w) \\
= & \left(\log \frac{2}{1-|a|^{2}}\right)^{p} \int_{\mathbb{D}}\left(f^{*}\left(\varphi_{a}(w)\right)\right)^{p}\left(1-\left|\varphi_{a}(w)\right|^{2}\right)^{\alpha p}\left(\log \frac{1}{|w|}\right)^{s} \frac{1}{\left(1-|w|^{2}\right)^{2}} d A(w) \\
\leq & \|f\|_{\mathcal{B}_{\alpha, \log }^{*}}^{p}\left(\log \frac{2}{1-|a|^{2}}\right)^{p} \int_{\mathbb{D}}\left(\log \frac{1}{|w|}\right)^{s}\left(1-|w|^{2}\right)^{-2} d A(w) \\
= & C(s, 2)|| f \|_{\mathcal{B}_{\alpha, \log }^{*}}^{p}
\end{aligned}
$$

By lemma 2.2, $C(s, 2)=\int_{\mathbb{D}}\left(\log \frac{1}{|w|}\right)^{s}\left(1-|w|^{2}\right)^{-2} d A(w)<\infty, \quad$ for $1<s<\infty$.

Thus, the quantity (D) is less than or equal to a constant times the quantity (A). Hence, it is proved.

Let us we give the following equivalent definition for $F_{\log }^{*}(p, q, s)$.

Definition 7. For $0<p, s<\infty,-2<q<\infty, \alpha=\frac{q+2}{p}$ and $q+s>-1$, a function $f \in H(\mathbb{D})$ is said to belong to $F_{\log }^{*}(p, q, s)$, if

$$
\sup _{a \in \mathbb{D}}\left(\log \frac{2}{1-|a|^{2}}\right)^{p} \int_{\mathbb{D}}\left(f^{*}(z)\right)^{p}\left(1-|z|^{2}\right)^{\alpha p-2}\left(1-\left|\varphi_{a}(z)\right|^{2}\right)^{s} d A(z)<\infty .
$$

Definition 8. A composition operator $C_{\phi}: \mathcal{B}_{\alpha, \log }^{*} \rightarrow F_{\log }^{*}(p, q, s)$ is said to be bounded if there is a positive constant $C$ so that $\left\|C_{\phi} f\right\|_{F_{\log }^{*}}(p, q, s) \leq C\|f\|_{\mathcal{B}_{\alpha, \log }^{*}}$ for all $f \in \mathcal{B}_{p, \alpha}^{*}$.

Definition 9. A composition operator $C_{\phi}: \mathcal{B}_{\alpha, \log }^{*} \rightarrow F_{\log }^{*}(p, q, s)$ is said to be compact if it maps any ball in $\mathcal{B}_{p, \alpha}^{*}$ onto a precompact set in $F^{*}(p, q, s)$.

The following lemma follows by standard arguments similar to those outline in [13. Hence, we omit the proof.

Lemma 2.3. Assume $\phi$ is a holomorphic mapping from $\mathbb{D}$ into itself. Let $0<p, s, \alpha<\infty,-2<$ $q<\infty$, then $C_{\phi}: \mathcal{B}_{\alpha, \log }^{*} \rightarrow F_{\log }^{*}(p, q, s)$ is compact if and only if for any bounded sequence $\left\{f_{n}\right\}_{n \in N} \in \mathcal{B}_{\alpha, \log }^{*}$ which converges to zero uniformly on compact subsets of $\mathbb{D}$ as $n \rightarrow \infty$ we have $\lim _{n \rightarrow \infty}\left\|C_{\phi} f_{n}\right\|_{F_{\log }^{*}(p, q, s)}=0$.

\section{$3 \quad$ D-metric space}

Topological properties of generalized metric space called $D$ - metric space was introduced in [1, see for example, ([2] and [3]). This structure of $D$-metric space is quite different from a 2-metric space and natural generalization of an ordinary metric space in some sense. 
Definition 10. [4] Let $X$ denote a nonempty set and $\mathbb{R}$ the set of real numbers. A function $D: X \times X \times X \rightarrow \mathbb{R}$ is said to be a D-metric on $X$ if it satisfies the following properties:

(i) $D(x, y, z) \geq 0$ for all $x, y, z \in X$ and equality holds if and only if $x=y=z$ (nonnegativity),

(ii) $D(x, y, z)=D(x, z, y)=\cdots($ symmetry),

(iii) $D(x, y, z) \leq D(x, y, a)+D(x, a, z)+D(a, y, z)$ for all $x, y, z, a \in X$ (tetrahedral inequality).

A nonempty set $X$ together with a $D$-metric $D$ is called a $D$-metric space and is represented by $(X, D)$. The generalization of a $D$-metric space with $D$-metric as a function of $n$ variables is provided in Dhage [2].

Example1.1: 4] Let $(X, d)$ be an ordinary metric space and define a function $D_{1}$ on $X^{3}$ by

$$
D_{1}(x, y, z)=\max \{d(x, y), d(y, z), d(z, x)\},
$$

for all $x, y, z \in X$. Then, the function $D_{1}$ is a $D$-metric on $X$ and $\left(X, D_{1}\right)$ is a $D$-metric space.

Example1.2: 4] Let $(X, d)$ be an ordinary metric space and define a function $D_{2}$ on $X^{3}$ by

$$
D_{2}(x, y, z)=d(x, y)+d(y, z)+d(z, x)
$$

for $x, y, z \in X$. Then, $D_{2}$ is a metric on $X$ and $\left(X, D_{2}\right)$ is a $D$-metric space.

Remark 1. Geometrically, the D-metric $D_{1}$ represents the diameter of a set consisting of three points $x, y$ and $z$ in $X$ and the $D$-metric $D_{2}(x, y, z)$ represents the perimeter of a triangle formed by three points $x, y, z$ in $X$ as its vertices.

Definition 11. (Cauchy sequence, completeness) [10] For every $m, n>N$. A sequence $\left(x_{n}\right)$ in a metric space $X=(X, d)$ is said to be-Cauchy if for every $\varepsilon>0$ there is an $N=N(\varepsilon)$ such that

$$
d\left(x_{m}, x_{n}\right)<\varepsilon .
$$

The space $X$ is said to be complete if every Cauchy sequence in $X$ converges (that is, has a limit which is an element of $X$ ).

The following theorem can be found in 4 :

Theorem 2. 4] Let d be an ordinary metric on $X$ and let $D_{1}$ and $D_{2}$ be corresponding associated $D$-metrics on $X$. Then, $\left(X, D_{1}\right)$ and $\left(X, D_{2}\right)$ are complete if and only if $(X, d)$ is complete. 


\section{$4 \quad D$-metrics in $\mathcal{B}_{\alpha, \log }^{*}$ and $F_{\log }^{*}(p, q, s)$}

In this section, we introduce a $D$-metric on $\mathcal{B}_{\alpha, \log }^{*}$ and $F_{\log }^{*}(p, q, s)$.

Let $0<p, s<\infty,-2<q<\infty$, and $0<\alpha<1$. First, we can find a $D$-metric in $\mathcal{B}_{\alpha, \log }^{*}$, for $f, g, h \in \mathcal{B}_{\alpha, \log }^{*}$ by defining

$$
\begin{aligned}
D\left(f, g, h ; \mathcal{B}_{\alpha, \log }^{*}\right):= & D_{\mathcal{B}_{\alpha, \log }^{*}}(f, g, h)+|| f-g\left\|_{\mathcal{B}_{\alpha, \log }}+\right\| g-h\left\|_{\mathcal{B}_{\alpha, \log }}+\right\| h-f \|_{\mathcal{B}_{\alpha, \log }} \\
& +|f(0)-g(0)|+|g(0)-h(0)|+|h(0)-f(0)|,
\end{aligned}
$$

where

$$
D_{\mathcal{B}_{\alpha, \log }^{*}}(f, g, h):=d_{\mathcal{B}_{\alpha, \log }^{*}}(f, g)+d_{\mathcal{B}_{\alpha, \log }^{*}}(g, h)+d_{\mathcal{B}_{\alpha, \log }^{*}}(h, f)
$$

and

$$
\begin{aligned}
D_{\mathcal{B}_{\alpha, \log }^{*}}(f, g, h):=\left(\sup _{z \in \mathbb{D}}\left|f^{*}(z)-g^{*}(z)\right|+\sup _{z \in \mathbb{D}}\left|g^{*}(z)-h^{*}(z)\right|+\sup _{z \in \mathbb{D}}\left|h^{*}(z)-f^{*}(z)\right|\right) \\
\times\left(\left(1-|z|^{2}\right)^{\alpha}\left(\log \frac{2}{1-|z|^{2}}\right)\right) .
\end{aligned}
$$

Also, for $f, g, h \in F_{\log }^{*}(p, q, s)$ we introduce a $D$-metric on $F_{\log }^{*}(p, q, s)$ by defining

$$
\begin{gathered}
D\left(f, g, h ; F_{\log }^{*}(p, q, s)\right):=D_{F_{\log }^{*}(p, q, s)}(f, g, h)+\|f-g\|_{F_{\log }(p, q, s)}+\|g-h\|_{F_{\log (p, q, s)}}+ \\
\|h-f\|_{F_{\log (p, q, s)}}+|f(0)-g(0)|+|g(0)-h(0)|+|h(0)-f(0)|,
\end{gathered}
$$

where

$$
D_{F_{\log }^{*}(p, q, s)}(f, g, h):=d_{F_{\log }^{*}(p, q, s)}(f, g)+d_{F_{\log }^{*}(p, q, s)}(g, h)+d_{F_{\log }^{*}(p, q, s)}(h, f)
$$

and

$$
d_{F_{\log }^{*}(p, q, s)}(f, g):=\left(\sup _{z \in \mathbb{D}} \ell^{p}(a) \int_{\mathbb{D}}\left|f^{*}(z)-g^{*}(z)\right|^{p}\left(1-|z|^{2}\right)^{q}\left(1-|\varphi(z)|^{2}\right)^{s} d A(z)\right)^{\frac{1}{p}} .
$$

Proposition 1. The class $\mathcal{B}_{\alpha, \log }^{*}$ equipped with the $D$-metric $D\left(., . ; \mathcal{B}_{\alpha, \log }^{*}\right)$ is a complete metric space. Moreover, $\mathcal{B}_{\alpha, \log , 0}^{*}$ is a closed (and therefore complete) subspace of $\mathcal{B}_{\alpha, \log }^{*}$.

Proof. Let $f, g, h, a \in \mathcal{B}_{\alpha, \log }^{*}$. Then, clearly

(i) $D\left(f, g, h ; \mathcal{B}_{\alpha, \log }^{*}\right) \geq 0$, for all $f, g, h \in \mathcal{B}_{\alpha, \log }^{*}$ 
(ii) $D\left(f, g, h ; \mathcal{B}_{\alpha, \log }^{*}\right)=D\left(f, h, g ; \mathcal{B}_{\alpha, \log }^{*}\right)=D\left(g, h, f ; \mathcal{B}_{\alpha, \log }^{*}\right)$.

(iii) $D\left(f, g, h ; \mathcal{B}_{\alpha, \log }^{*}\right) \leq D\left(f, g, a ; \mathcal{B}_{\alpha, \log }^{*}\right)+D\left(f, a, h ; \mathcal{B}_{\alpha, \log }^{*}\right)+D\left(a, g, h ; \mathcal{B}_{\alpha, \log }^{*}\right)$

for all $f, g, h, a \in \mathcal{B}_{\alpha, \log }^{*}$

(iv) $D\left(f, g, h ; \mathcal{B}_{\alpha, \log }^{*}\right)=0$ implies $f=g=h$.

Hence, $D$ is a $D$-metric on $\mathcal{B}_{\alpha, \log }^{*}$, and $\left(\mathcal{B}_{\alpha, \log }^{*}, D\right)$ is $D$-metric space.

To prove the completeness, we use Theorem 2 let $\left(f_{n}\right)_{n=1}^{\infty}$ be a Cauchy sequence in the metric space $\left(\mathcal{B}_{\alpha, \log }^{*}, d\right)$, that is, for any $\varepsilon>0$ there is an $N=N(\varepsilon) \in \mathbb{N}$ such that $d\left(f_{n}, f_{m} ; \mathcal{B}_{\alpha, \log }^{*}\right)<\varepsilon$, for all $n, m>N$. Since $\left(f_{n}\right) \subset B(\mathbb{D})$, the family $\left(f_{n}\right)$ is uniformly bounded and hence normal in $\mathbb{D}$. Therefore, there exists $f \in B(\mathbb{D})$ and a subsequence $\left(f_{n_{j}}\right)_{j=1}^{\infty}$ such that $f_{n_{j}}$ converges to $f$ uniformly on compact subsets of $\mathbb{D}$. It follows that $f_{n}$ also converges to $f$ uniformly on compact subsets, and by the Cauchy formula, the same also holds for the derivatives. Now let $m>N$. Then, the uniform convergence yields

$$
\begin{aligned}
& \left|f^{*}(z)-f_{m}^{*}(z)\right|\left(1-|z|^{2}\right)^{\alpha}\left(\log \frac{2}{1-|z|^{2}}\right) \\
= & \lim _{n \rightarrow \infty}\left|f_{n}^{*}(z)-f_{m}^{*}(z)\right|\left(1-|z|^{2}\right)^{\alpha}\left(\log \frac{2}{1-|z|^{2}}\right) \\
\leq & \lim _{n \rightarrow \infty} d\left(f_{n}, f_{m} ; \mathcal{B}_{\alpha, \log }^{*}\right) \leq \varepsilon
\end{aligned}
$$

for all $z \in \mathbb{D}$, and it follows that $\|f\|_{\mathcal{B}_{\alpha, \log }^{*}} \leq\left\|f_{m}\right\|_{\mathcal{B}_{\alpha, \log }^{*}}+\varepsilon$. Thus $f \in \mathcal{B}_{\alpha, \log }^{*}$ as desired. Moreover, the above inequality and the compactness of the usual $\mathcal{B}_{\alpha \text {, log }}^{*}$ space imply that $\left(f_{n}\right)_{n=1}^{\infty}$ converges to $f$ with respect to the metric $d$, and $\left(\mathcal{B}_{\alpha, \log }^{*}, D\right)$ is complete $D$-metric space.

Since $\lim _{n \rightarrow \infty} d\left(f_{n}, f_{m} ; \mathcal{B}_{\alpha, \log }^{*}\right) \leq \varepsilon$, the second part of the assertion follows.

Next we give characterization of the complete $D$-metric space $D\left(., . ; F_{\log }^{*}(p, q, s)\right)$.

Proposition 2. The class $F_{\log }^{*}(p, q, s)$ equipped with the D-metric $D\left(.,, ; F_{\log }^{*}(p, q, s)\right)$ is a complete metric space. Moreover, $F_{\log , 0}^{*}(p, q, s)$ is a closed (and therefore complete) subspace of $F_{\log }^{*}(p, q, s)$.

Proof. Let $f, g, h, a \in F_{\log }^{*}(p, q, s)$. Then clearly

(i) $D\left(f, g, h ; F_{\log }^{*}(p, q, s)\right) \geq 0$, for all $f, g, h \in F_{\log }^{*}(p, q, s)$.

(ii) $D\left(f, g, h ; F_{\log }^{*}(p, q, s)\right)=D\left(f, h, g ; F_{\log }^{*}(p, q, s)\right)=D\left(g, h, f ; F_{\log }^{*}(p, q, s)\right)$. 
(iii) $D\left(f, g, h ; F_{\log }^{*}(p, q, s)\right) \leq D\left(f, g, a ; F_{\log }^{*}(p, q, s)\right)+D\left(f, a, h ; F_{\log }^{*}(p, q, s)\right)$

$$
+D\left(a, g, h ; F_{\log }^{*}(p, q, s)\right)
$$

for all $f, g, h, a \in F_{\log }^{*}(p, q, s)$.

(iv) $D\left(f, g, h ; F_{\log }^{*}(p, q, s)\right)=0$ implies $f=g=h$.

Hence, $D$ is a $D$-metric on $F_{\log }^{*}(p, q, s)$, and $\left(F_{\log }^{*}(p, q, s), D\right)$ is $D$-metric space.

For the complete proof, by using Theorem 2] let $\left(f_{n}\right)_{n=1}^{\infty}$ be a Cauchy sequence in the metric space $\left(F_{\log }^{*}(p, q, s), d\right)$, that is, for any $\varepsilon>0$ there is an $N=N(\varepsilon) \in \mathbb{N}$ so that $d\left(f_{n}, f_{m} ; F_{\log }^{*}(p, q, s)\right)<$ $\varepsilon$, for all $n, m>N$. Since $\left(f_{n}\right) \subset B(\mathbb{D})$, such that $f_{n_{j}}$ converges to $f$ uniformly on compact subsets of $\mathbb{D}$. It follows that $f_{n}$ also converges to $f$ uniformly on compact subsets, now let $m>N$, and $0<r<1$. Then, the Fatou's yields

$$
\begin{aligned}
& \int_{\mathbb{D}(0, r)}\left|f^{*}(z)-f_{m}^{*}(z)\right|^{p}\left(1-|z|^{2}\right)^{q}\left(1-\left|\varphi_{a}(z)\right|^{2}\right)^{s} d A(z) \\
= & \int_{\mathbb{D}(0, r)} \lim _{n \rightarrow \infty}\left|f_{n}^{*}(z)-f_{m}^{*}(z)\right|^{p}\left(1-|z|^{2}\right)^{q}\left(1-\left|\varphi_{a}(z)\right|^{2}\right)^{s} d A(z) \\
\leq & \lim _{n \rightarrow \infty} \int_{\mathbb{D}(0, r)}\left|f^{*}(z)-f_{m}^{*}(z)\right|^{p}\left(1-|z|^{2}\right)^{q}\left(1-\left|\varphi_{a}(z)\right|^{2}\right)^{s} d A(z) \leq \varepsilon^{p},
\end{aligned}
$$

and by taking $r \rightarrow 1^{-}$, it follows that,

$$
\begin{gathered}
\int_{\mathbb{D}}\left(f^{*}(z)\right)^{p}\left(1-|z|^{2}\right)^{q}\left(1-\left|\varphi_{a}(z)\right|^{2}\right)^{s} d A(z) \\
\leq 2^{p} \varepsilon^{p}+2^{p} \int_{\mathbb{D}}\left(f_{m}^{*}(z)\right)^{p}\left(1-|z|^{2}\right)^{q}\left(1-\left|\varphi_{a}(z)\right|^{2}\right)^{s} d A(z) .
\end{gathered}
$$

This yields

$$
\|f\|_{F_{\log }^{*}(p, q, s)}^{p} \leq 2^{p}\left\|f_{m}\right\|_{F_{\log }^{*}(p, q, s)}^{p}+2^{p} \varepsilon^{p}
$$

And thus $f \in F_{\log }^{*}(p, q, s)$. We also find that $f_{n} \rightarrow f$ with respect to the metric of $\left(F_{\log }^{*}(p, q, s), D\right)$ and $\left(F_{\log }^{*}(p, q, s), D\right)$ is complete $D$-metric space. The second part of the assertion follows.

\section{Composition operators of $C_{\phi}: \mathcal{B}_{\alpha, \log }^{*} \rightarrow F_{\log }^{*}(p, q, s)$}

In this section, we study boundedness and compactness of composition operators on $\mathcal{B}_{\alpha, \log }^{*}$ and $F_{\log }^{*}(p, q, s)$ spaces. We need the following notation:

$$
\Phi_{\phi}(\alpha, p, s ; a)=\ell^{p}(a) \int_{\mathbb{D}}\left|\phi^{\prime}(z)\right|^{p} \frac{\left(1-|z|^{2}\right)^{\alpha p-2}\left(1-\left|\varphi_{a}(z)\right|^{2}\right)^{s}}{\left(1-|\phi(z)|^{2}\right)^{\alpha p}\left(\log \frac{2}{\left(1-|\phi(z)|^{2}\right)}\right)^{p}} d A(z)
$$


where $\ell^{p}(a)=\left(\log \frac{2}{1-|a|^{2}}\right)^{p}$.

For $0<\alpha<1$, we suppose there exist two functions $f, g \in \mathcal{B}_{\alpha, \log }^{*}$ such that for some constant C,

$$
\left(\left|f^{*}(z)\right|+\left|g^{*}(z)\right|\right) \geq \frac{C}{\left(1-|z|^{2}\right)^{\alpha}\left(\log \frac{2}{1-|a|^{2}}\right)^{p}}>0, \quad \text { for each } z \in \mathbb{D} .
$$

Now, we provide the following theorem:

Theorem 3. Assume $\phi$ is a holomorphic mapping from $\mathbb{D}$ into itself and let $0<p, 1<s<\infty, 0<$ $\alpha \leq 1$. Then the induced composition operator $C_{\phi}$ maps $\mathcal{B}_{\alpha, \log }^{*}$ into $F_{\log }^{*}(p, \alpha p-2, s)$ is bounded if and only if,

$$
\sup _{z \in \mathbb{D}} \Phi_{\phi}(\alpha, p, s ; a)<\infty
$$

Proof. First assume that $\sup _{z \in \mathbb{D}} \Phi_{\phi}(\alpha, p, s ; a)<\infty$ is held, and $f \in \mathcal{B}_{\alpha, \log }^{*}$ with $\|f\|_{\mathcal{B}_{\alpha, \log }} \leq 1$, we can see that

$$
\begin{aligned}
& \left\|C_{\phi} f\right\|_{F_{\log }^{*}(p, \alpha p-2, s)}^{p} \\
= & \sup _{a \in \mathbb{D}} \ell^{p}(a) \int_{\mathbb{D}}\left((f \circ \phi)^{*}(z)\right)^{p}\left(1-|z|^{2}\right)^{\alpha p-2}\left(1-\left|\varphi_{a}(z)\right|^{2}\right)^{s} d A(z) \\
= & \sup _{a \in \mathbb{D}} \ell^{p}(a) \int_{\mathbb{D}}\left(f^{*}(\phi(z))\right)^{p}\left|\phi^{\prime}(z)\right|^{\alpha p-2}\left(1-\left|\varphi_{a}(z)\right|^{2}\right)^{s} d A(z) \\
\leq & \|f\|_{\mathcal{B}_{\alpha, \log }^{*}}^{p} \sup _{a \in \mathbb{D}} \ell^{p}(a) \int_{\mathbb{D}} \frac{\left|\phi^{\prime}(z)\right|^{p}\left(1-|z|^{2}\right)^{\alpha p-2}\left(1-\left|\varphi_{a}(z)\right|^{2}\right)^{s}}{\left(1-\left|\phi(z)^{2}\right|\right)^{p \alpha}\left(\log \frac{2}{1-|z|^{2}}\right)} d A(z) \\
= & \|f\|_{\mathcal{B}_{\alpha, \log }^{*}}^{p} \Phi_{\phi}(\alpha, p, s ; a)<\infty .
\end{aligned}
$$

For the other direction, we use the fact that for each function $f \in \mathcal{B}_{\alpha, \log }^{*}$, the analytic function 
$C_{\phi}(f) \in F_{\log }^{*}(p, \alpha p-2, s)$. Then, using the functions of lemma 1.2

$$
\begin{aligned}
& 2^{p}\left\{\left\|C_{\phi} f_{1}\right\|_{F_{\log }^{*}(p, \alpha p-2, s)}^{p}+|| C_{\phi} f_{2} \|_{F_{\log }^{*}(p, \alpha p-2, s)}^{p}\right\} \\
= & 2^{p}\left\{\sup _{a \in \mathbb{D}} \ell^{p}(a) \int_{\mathbb{D}}\left[\left(\left(f_{1} \circ \phi\right)^{*}(z)\right)^{p}+\left(\left(f_{2} \circ \phi\right)^{*}(z)\right)^{p}\right]\right. \\
& \left.\times\left(1-|z|^{2}\right)^{\alpha p-2}\left(1-\left|\varphi_{a}(z)\right|^{2}\right)^{s} d A(z)\right\} \\
\geq & \left\{\sup _{a \in \mathbb{D}} \ell^{p}(a) \int_{\mathbb{D}}\left[\left(f_{1} \circ \phi\right)^{*}(z)+\left(f_{2} \circ \phi\right)^{*}(z)\right]^{p}\right. \\
& \left.\times\left(1-|z|^{2}\right)^{\alpha p-2}\left(1-\left|\varphi_{a}(z)\right|^{2}\right)^{s} d A(z)\right\} \\
\geq & \left\{\sup _{a \in \mathbb{D}} \ell^{p}(a) \int_{\mathbb{D}}\left[\left(f_{1}^{*}(\phi)\right)(z)+\left(f_{2}^{*}(\phi)\right)(z)\right]^{p}\right. \\
& \left.\times\left|\phi^{\prime}(z)\right|^{p}\left(1-|z|^{2}\right)^{\alpha p-2}\left(1-\left|\varphi_{a}(z)\right|^{2}\right)^{s} d A(z)\right\} \\
\geq & C\left\{\sup _{a \in \mathbb{D}} \ell^{p}(a) \int_{\mathbb{D}}\left|\phi^{\prime}(z)\right|^{p} \frac{\left(1-|z|^{2}\right)^{\alpha p-2}\left(1-\left|\varphi_{a}(z)\right|^{2}\right)^{s}}{\left(1-|\phi(z)|^{2}\right)^{\alpha p}\left(\log \frac{2}{\left(1-|\phi(z)|^{2}\right)}\right)^{p}} d A(z)\right\} \\
\geq & C \sup _{a \in \mathbb{D}} \Phi_{\phi}(\alpha, p, s ; a) .
\end{aligned}
$$

Hence $C_{\phi}$ is bounded, the proof is completed.

The composition operator $C_{\phi}: \mathcal{B}_{\alpha, \log }^{*} \rightarrow F_{\log }^{*}(p, \alpha p-2, s)$ is compact if and only if for every sequence $f_{n} \in \mathbb{N} \subset F_{\log }^{*}(p, \alpha p-2, s)$ is bounded in $F_{\log }^{*}(p, \alpha p-2, s)$ norm and $f_{n} \rightarrow 0, n \rightarrow \infty$, uniformly on compact subset of the unit disk (where $\mathbb{N}$ be the set of all natural numbers), hence,

$$
\left\|C_{\phi}\left(f_{n}\right)\right\|_{F_{\log }^{*}(p, \alpha p-2, s)} \rightarrow 0, n \rightarrow \infty .
$$

Now, we describe compactness in the following result:

Theorem 4. Let $0<p, 1<s<\infty, \alpha<\infty$. If $\phi$ is an analytic self-map of the unit disk, then the induced composition operator $C_{\phi}: \mathcal{B}_{\alpha, \log }^{*} \rightarrow F_{\log }^{*}(p, \alpha p-2, s)$ is compact if and only if $\phi \in F_{\log }^{*}(p, \alpha p-2, s)$, and

$$
\lim _{r \rightarrow 1} \sup _{a \in \mathbb{D}} \Phi_{\phi}(\alpha, p, s ; a) \rightarrow 0
$$

Proof. Let $C_{\phi}: \mathcal{B}_{\alpha, \log }^{*} \rightarrow F_{\log }^{*}(p, \alpha p-2, s)$ be compact. This means that $\phi \in F_{\log }^{*}(p, \alpha p-2, s)$.

Let

$$
U_{r}^{1}=\{z:|\phi(z)|>r, r \in(0,1)\}
$$


and

$$
U_{r}^{2}=\{z:|\phi(z)| \leq r, r \in(0,1)\} .
$$

Let $f_{n}(z)=\frac{z^{n}}{n}$ if $\alpha \in[0, \infty)$ or $f_{n}(z)=\frac{z^{n}}{n^{1-\alpha}}$ if $\alpha \in(0,1)$. Without loss of generality, we only consider $\alpha \in(0,1)$. Since $\left\|f_{n}\right\|_{\mathcal{B}_{\alpha, \log }^{*}} \leq M$ and $f_{n}(z) \rightarrow 0$ as $n \rightarrow \infty$, locally uniformly on the unit disk, then $\left\|C_{\phi}\left(f_{n}\right)\right\|_{F_{\log }^{*}(p, \alpha p-2, s)}, n \rightarrow \infty$. This means that for each $r \in(0,1)$ and for all $\varepsilon>0$, there exist $N \in \mathbb{N}$ so that if $n \geq N$, then

$$
\frac{N^{\alpha p}}{r^{p(1-N)}} \sup _{a \in \mathbb{D}} \ell^{p}(a) \int_{U_{r}^{1}}\left|\phi^{\prime}(z)\right|^{p}\left(1-|z|^{2}\right)^{\alpha p-2}\left(1-\left|\varphi_{a}(z)\right|^{2}\right)^{s} d A(z)<\varepsilon .
$$

If we choose $r$ so that $\frac{N^{\alpha p}}{r^{p(1-N)}}=1$, then

$$
\sup _{a \in \mathbb{D}} \ell^{p}(a) \int_{U_{r}^{1}}\left|\phi^{\prime}(z)\right|^{p}\left(1-|z|^{2}\right)^{\alpha p-2}\left(1-\left|\varphi_{a}(z)\right|^{2}\right)^{s} d A(z)<\varepsilon .
$$

Let now $f$ be with $\|f\|_{\mathcal{B}_{\alpha, \log }^{*}} \leq 1$. We consider the functions $f_{t}(z)=f(t z), t \in(0,1) . f_{t} \rightarrow f$ uniformly on compact subset of the unit disk as $t \rightarrow 1$ and the family $\left(f_{t}\right)$ is bounded on $\mathcal{B}_{\alpha, \log }^{*}$, thus

$$
\left\|\left(f_{t} \circ \phi\right)-(f \circ \phi)\right\| \rightarrow 0 .
$$

Due to compactness of $C_{\phi}$, we get that for $\varepsilon>0$ there is $t \in(0,1)$ so that

$$
\sup _{a \in \mathbb{D}} \ell^{p}(a) \int_{\mathbb{D}}\left|F_{t}(\phi(z))\right|^{p}\left(1-|z|^{2}\right)^{\alpha p-2}\left(1-\left|\varphi_{a}(z)\right|^{2}\right)^{s} d A(z)<\varepsilon,
$$

where

$$
F_{t}(\phi(z))=\left[(f \circ \phi)^{*}-\left(f_{t} \circ \phi\right)^{*}\right]
$$

Thus, if we fix $t$, then

$$
\begin{aligned}
& \sup _{a \in \mathbb{D}} \ell^{p}(a) \int_{U_{r}^{1}}\left((f \circ \phi)^{*}(z)\right)^{p}\left(1-|z|^{2}\right)^{\alpha p-2}\left(1-\left|\varphi_{a}(z)\right|^{2}\right)^{s} d A(z) \\
\leq & 2^{p} \sup _{a \in \mathbb{D}} \ell^{p}(a) \int_{U_{r}^{1}}\left|F_{t}(\phi(z))\right|^{p}\left(1-|z|^{2}\right)^{\alpha p-2}\left(1-\left|\varphi_{a}(z)\right|^{2}\right)^{s} d A(z) \\
& +2^{p} \sup _{a \in \mathbb{D}} \ell^{p}(a) \int_{U_{r}^{1}}\left(\left(f_{t} \circ \phi\right)^{*}(z)\right)^{p}\left(1-|z|^{2}\right)^{\alpha p-2}\left(1-\left|\varphi_{a}(z)\right|^{2}\right)^{s} d A(z) \\
\leq & 2^{p} \varepsilon+\|\left. f_{t}^{*}\right|_{H^{\infty}} ^{p} \sup _{a \in \mathbb{D}} \ell^{p}(a) \int_{U_{r}^{1}}\left|\phi^{\prime}(z)\right|^{p}\left(1-|z|^{2}\right)^{\alpha p-2}\left(1-\left|\varphi_{a}(z)\right|^{2}\right)^{s} d A(z) \\
\leq & 2^{p} \varepsilon+2^{p} \varepsilon\left\|f_{t}^{*}\right\|_{H^{\infty}}^{p} .
\end{aligned}
$$


i.e,

$$
\begin{aligned}
& \sup _{a \in \mathbb{D}} \ell^{p}(a) \int_{U_{r}^{1}}\left((f \circ \phi)^{*}(z)\right)^{p}\left(1-|z|^{2}\right)^{\alpha p-2}\left(1-\left|\varphi_{a}(z)\right|^{2}\right)^{s} d A(z) \\
\leq & 2^{p} \varepsilon\left(1+\left\|f_{t}^{*}\right\|_{H^{\infty}}^{p}\right),
\end{aligned}
$$

where we have used (4). On the other hand, for each $\|f\|_{\mathcal{B}_{\alpha, \log }^{*}} \leq 1$ and $\varepsilon>0$, there exists a $\delta$ depending on $f$ and $\varepsilon$, so that for $r \in[\delta, 1)$,

$$
\sup _{a \in \mathbb{D}} \ell^{p}(a) \int_{U_{r}^{1}}\left((f \circ \phi)^{*}(z)\right)^{p}\left(1-|z|^{2}\right)^{\alpha p-2}\left(1-\left|\varphi_{a}(z)\right|^{2}\right)^{s} d A(z)<\varepsilon
$$

Since $C_{\phi}$ is compact, then it maps the unit ball of $\mathcal{B}_{\alpha, \log }^{*}$ to a relatively compact subset of $F_{\log }^{*}(p, q, s)$. Thus, for each $\varepsilon>0$, there exists a finite collection of functions $f_{1}, f_{2}, \ldots, f_{n}$ in the unit ball of $\mathcal{B}_{\alpha, \log }^{*}$ so that for each $\|f\|_{\mathcal{B}_{\alpha, \log }^{*}}$, there is $k \in\{1,2,3, \ldots, n\}$ so that

$$
\sup _{a \in \mathbb{D}} \ell^{p}(a) \int_{U_{r}^{1}}\left|F_{k}(\phi(z))\right|^{p}\left(1-|z|^{2}\right)^{\alpha p-2}\left(1-\left|\varphi_{a}(z)\right|^{2}\right)^{s} d A(z)<\varepsilon
$$

where

$$
F_{k}(\phi(z))=\left[(f \circ \phi)^{*}-\left(f_{k} \circ \phi\right)^{*}\right]
$$

Also, using (5), we get for $\delta=\max _{1 \leq k \leq n} \delta\left(f_{k}, \varepsilon\right)$ and $r \in[\delta, 1)$, that

$$
\sup _{a \in \mathbb{D}} \ell^{p}(a) \int_{U_{r}^{1}}\left(\left(f_{k} \circ \phi\right)^{*}(z)\right)^{p}\left(1-|z|^{2}\right)^{\alpha p-2}\left(1-\left|\varphi_{a}(z)\right|^{2}\right)^{s} d A(z)<\varepsilon .
$$

Hence, for any $f,\|f\|_{\mathcal{B}_{\alpha, \log }^{*}} \leq 1$, combining the two relations as above, we get the following

$$
\sup _{a \in \mathbb{D}} \ell^{p}(a) \int_{U_{r}^{1}}\left((f \circ \phi)^{*}(z)\right)^{p}\left(1-|z|^{2}\right)^{\alpha p-2}\left(1-\left|\varphi_{a}(z)\right|^{2}\right)^{s} d A(z) \leq 2^{p} \varepsilon
$$

Therefore, we get that (2) holds. For the sufficiency, we use that $\phi \in F_{\log }^{*}(p, \alpha p-2, s)$ and (2) holds.

Let $\left\{f_{n}\right\}_{n \in \mathbb{N}}$ be a sequence of functions in the unit ball of $\mathcal{B}_{\alpha, \log }^{*}$ so that $f_{n} \rightarrow 0$ as $n \rightarrow \infty$, uniformly on the compact subsets of the unit disk. Let also $r \in(0,1)$. Then,

$$
\begin{aligned}
& \quad \| f_{n} \circ \phi||_{F_{\log }^{*}(p, \alpha p-2, s)}^{p} \leq 2^{p}\left|f_{n}(\phi(0))\right| \\
& +2^{p} \sup _{a \in \mathbb{D}} \ell^{p}(a) \int_{U_{r}^{2}}\left(\left(f_{n} \circ \phi\right)^{*}(z)\right)^{p}\left(1-|z|^{2}\right)^{\alpha p-2}\left(1-\left|\varphi_{a}(z)\right|^{2}\right)^{s} d A(z) \\
& +2^{p} \sup _{a \in \mathbb{D}} \ell^{p}(a) \int_{U_{r}^{1}}\left(\left(f_{n} \circ \phi\right)^{*}(z)\right)^{p}\left(1-|z|^{2}\right)^{\alpha p-2}\left(1-\left|\varphi_{a}(z)\right|^{2}\right)^{s} d A(z) \\
& =2^{p}\left(I_{1}+I_{2}+I_{3}\right) .
\end{aligned}
$$


Since $f_{n} \rightarrow 0$ as $n \rightarrow \infty$, locally uniformly on the unit disk, then $I_{1}=\left|f_{n}(\phi(0))\right|$ goes to zero as $n \rightarrow \infty$ and for each $\varepsilon>0$, there is $N \in \mathbb{N}$ so that for each $n>N$,

$$
\begin{aligned}
& I_{2}=\sup _{a \in \mathbb{D}} \ell^{p}(a) \int_{U_{r}^{2}}\left(\left(f_{n} \circ \phi\right)^{*}(z)\right)^{p}\left(1-|z|^{2}\right)^{\alpha p-2}\left(1-\left|\varphi_{a}(z)\right|^{2}\right)^{s} d A(z) \\
\leq & \varepsilon\|\phi\|_{F_{\log }^{*}(p, \alpha p-2, s)}^{p} .
\end{aligned}
$$

We also observe that

$$
\begin{aligned}
& I_{3}=\sup _{a \in \mathbb{D}} \ell^{p}(a) \int_{U_{r}^{1}}\left(\left(f_{n} \circ \phi\right)^{*}(z)\right)^{p}\left(1-|z|^{2}\right)^{\alpha p-2}\left(1-\left|\varphi_{a}(z)\right|^{2}\right)^{s} d A(z) \\
\leq & \|f\|_{\mathcal{B}_{\alpha, \log }^{*}} \\
& \times \sup _{a \in \mathbb{D}} \ell^{p}(a) \int_{U_{r}^{1}}\left|\phi^{\prime}(z)\right|^{p} \frac{\left(1-|z|^{2}\right)^{\alpha p-2}\left(1-\left|\varphi_{a}(z)\right|^{2}\right)^{s}}{\left(1-|\phi(z)|^{2}\right)^{\alpha p}\left(\log \frac{2}{\left(1-|\phi(z)|^{2}\right)}\right)^{p}} d A(z) .
\end{aligned}
$$

Under the assumption that (2) holds, then for every $n>N$ and for every $\varepsilon>0$, there exists $r_{1}$ so that for every $r>r_{1}, I_{3}<\varepsilon$.

Thus, if $\phi(z) \in F_{\log }^{*}(p, \alpha p-2, s)$, we get

$$
\left\|f_{n} \circ \phi\right\|_{F_{\log }^{*}(p, \alpha p-2, s)}^{p} \leq 2^{p}\left\{0+\varepsilon\|\phi\|_{F_{\log }^{*}(p, \alpha p-2, s)}^{p}+\varepsilon\right\} \leq C \varepsilon .
$$

Combining the above, we get $\left\|C_{\phi}\left(f_{n}\right)\right\|_{F_{\log }^{*}(p, \alpha p-2, s)}^{p} \rightarrow 0$ as $n \rightarrow \infty$ which proves compactness. Thus, the theorem we presented is proved.

\section{Conclusions}

We have obtained some essential and important $D$-metric spaces. Moreover, the important properties for $D$-metric on $\mathcal{B}_{\alpha, \log }^{*}$ and $F_{\log }^{*}(p, q, s)$ are investigated in Section 4 . Finally, we introduced composition operators in hyperbolic weighted family of function spaces.

\section{Acknowledgements}

The authors would like to thank the referees for their useful comments, which improved the original manuscript. 


\section{References}

[1] B. C. Dhage, A study of some fixed point theorem. Ph.D. Thesis, Marathwada Univ. Aurangabad, India, 1984.

[2] B. C. Dhage, Generalized metric spaces and mappings with fixed point, Bull. Cal. Math. Soc, 84(4)(1992), 329-336.

[3] B. C. Dhage, On generalized metric spaces and topological structure. II, Pure Appl. Math. Sci. 40 (1994), no. 1-2, 37-41

[4] B. C. Dhage, Generalized metric spaces and topological structure. I, An. Ştiinţ. Univ. Al. I. Cuza Iaşi. Mat. (N.S.) 46 (2000), no. 1, 3-24 (2001).

[5] A. El-Sayed Ahmed and M. A. Bakhit, Composition operators acting between some weighted Möbius invariant spaces, Ann. Funct. Anal. 2 (2011), no. 2, 138-152.

[6] A. El-Sayed Ahmed, A. Kamal and T. I. Yassen, Characterizations for certain analytic functions by series expansions with Hadamard gaps, Cubo 16 (2014), no. 1, 81-93.

[7] T. Hosokawa, Differences of weighted composition operators on the Bloch spaces, Complex Anal. Oper. Theory 3 (2009), no. 4, 847-866.

[8] A Kamal, A El-Sayed Ahmed, and T. I. Yassen, Quasi-Metric Spaces and Composition Operators on $B *_{\alpha}, \log$ and $Q * p, \log$ Spaces, Journal of Computational and Theoretical Nanoscience 12 (2015), no. 8, 1795-1801.

[9] A Kamal and T. I. Yassen, Some properties of composition operator acting between general hyperbolic type spaces, International Journal of Mathematical Analysis and Applications 2 (2015), no. 2, 17-26

[10] E. Kreyszig, Introductory functional analysis with applications, John Wiley \& Sons, New York, 1978.

[11] F. Pérez-González, J. Rättyä and J. Taskinen, Lipschitz continuous and compact composition operators in hyperbolic classes, Mediterr. J. Math. 8 (2011), no. 1, 125-135.

[12] R. Zhao, On $\alpha$-Bloch functions and VMOA, Acta Math. Sci. (English Ed.) 16 (1996), no. 3, $349-360$.

[13] M. Tjani, Compact composition operators on Besov spaces, Trans. Amer. Math. Soc. 355 (2003), no. 11, 4683-4698.

[14] R. Zhao, On a general family of function spaces, Ann. Acad. Sci. Fenn. Math. Diss. No. 105 (1996), $56 \mathrm{pp}$. 
[15] R. Zhao, On logarithmic Carleson measures, Acta Sci. Math. (Szeged) 69 (2003), no. 3-4, 605-618. 